\title{
Regulation of pre-fusion events: recruitment of M-cadherin to microrafts organized at fusion-competent sites of myogenic cells
}

\author{
Atsushi Mukai and Naohiro Hashimoto*
}

\begin{abstract}
Background: Previous research indicates that the membrane ruffles and leading edge of lamellipodia of myogenic cells contain presumptive fusion sites. A micrometer-sized lipid raft (microraft) is organized at the presumptive fusion site of mouse myogenic cells in a cell-contact independent way and serves as a platform tethering adhesion proteins that are relevant to cell fusion. However, the mechanisms underlying recruitment of adhesion proteins to lipid rafts and microraft organization remain unknown.

Results: Here we show that small G-protein Rac1 was required for microraft organization and subsequent cell fusion. However, Rac1 activity was unnecessary for recruitment of M-cadherin to lipid rafts. We found that p120 catenin (p120) binds to M-cadherin exclusively in lipid rafts of differentiating myogenic cells. The Src kinase inhibitor SU6656 prevented p120 binding to M-cadherin and their recruitment to lipid rafts, then suppressed microraft organization, membrane ruffling, and myogenic cell fusion. Suppression of membrane ruffling in SU6656-treated cells was partially restored by pretreatment with the protein tyrosine phosphatase inhibitor vanadate. The present analyses using an antibody to tyrosine phosphorylated p120 suggest that Src family kinases play a role in binding of p120 to M-cadherin and the recruitment of M-cadherin to lipid rafts through phosphorylation of putative substrates other than p120.

Conclusions: The present study showed that the procedure establishing fusion-competent sites consists of two sequential events: recruitment of adhesion complexes to lipid rafts and organization of microrafts. The recruitment of M-cadherin to lipid rafts depended on interaction with p120 catenin, whereas the organization of microrafts was controlled by a small G protein, Rac1.
\end{abstract}

Keywords: Myogenesis, Cell fusion, M-cadherin, p120 catenin, Rac1, Tyrosine phosphorylation

\section{Background}

A skeletal muscle fiber is an extra-large, multinucleated, non-mitotic cells that are responsible for the generation of force by skeletal muscle under the control of motor neurones. This unique terminally differentiated cells are derived from multinucleated myotubes, which are formed by the fusion of mononucleated myogenic progenitor cells (myoblasts). Myoblasts are descendants of muscle stem cells called muscle satellite cells and show unique capacities, including multipotentiality [1] and the ability to fuse with each other in a cell-autonomous way. Myoblast

\footnotetext{
* Correspondence: nao@ncgg.go.jp

Department of Regenerative Medicine, National Institute for Longevity

Sciences, National Center for Geriatrics and Gerontology, 35 Gengo, Morioka, Oobu, Aichi 474-8522, Japan
}

fusion is cell-specific, because myoblasts do not fuse with non-myogenic cells, and essential for skeletal muscle development and repair.

Myoblast fusion consists of a series of steps: cell-cell contact, recognition, adhesion, and plasma membrane breakdown/union [2-4]. Plasma membrane breakdown/ union is initially induced in a discrete area of the plasma membrane [5,6]. Thus, specialization of presumptive fusion sites in the plasma membrane is prerequisite for myogenic cell fusion. Extracellular matrix receptor integrins and adhesion molecules such as cadherins, NCAM, CD9, CD81, and ADAMs might contribute to regulation of the recognition/adhesion steps of myoblast fusion [7-9]. However, how they accumulate at the discrete, presumptively

\section{Biomed Central}


fusion-competent sites of the plasma membrane remains to be determined.

Our previous study showed that the leading edge of lamellipodia and membrane ruffles of differentiating myogenic cells contain fusion-competent sites in the plasma membrane [5]. Adhesion proteins accumulate at the presumptive fusion sites of differentiating myogenic cells in a lipid raft-dependent fashion prior to cell contact [10], while membrane fusion takes place within cholesterol-free sites of the plasma membrane [11]. Membrane cholesterol is enriched in lipid rafts. However, these results are not discrepant because dynamic clustering and dispersion of lipid rafts plays a pivotal role in the redistribution of adhesion complexes and membrane cholesterol at the presumptively fusion-competent sites of the plasma membrane in myogenic cells [10]. The adhesion complexes accumulate in a micrometer-scaled lipid raft (microraft) in a cell contactindependent fashion under the differentiation-inducing condition, whereas they are distributed in both raft and non-raft fractions of plasma membranes in growing myogenic cells. Therefore, both the recruitment of adhesion complexes to lipid rafts and the organization of microrafts might be critical to plasma membrane breakdown/union of myogenic cells.

M-cadherin is a myogenic cell-specific classic cadherin that plays a pivotal role in myogenic cell fusion $[8,12-16]$. Adhesion-complex proteins including M-cadherin, $\beta$ catenin, and p120 catenin accumulate in microrafts at presumptive fusion sites even if myogenic cells do not contact a fusion partner [10]. The present study showed that recruitment of M-cadherin/p120 complex to lipid rafts and organization of microrafts are two distinct pre-fusion events that are essential for the specialization of fusioncompetent sites.

\section{Methods}

\section{Cell culture}

The mouse myogenic cell clone Ric10 was established from muscle satellite cells of the normal gastrocnemius muscle of an adult female ICR mouse [1,5]. Ric10 cells were plated on dishes coated with type I collagen (Sumilon, Tokyo, Japan) and cultured at $37^{\circ} \mathrm{C}$ under $10 \% \mathrm{CO}_{2}$ in pmGM consisting of Dulbecco's modified Eagle's medium supplemented with 20\% fetal bovine serum (FBS), $2 \%$ Ultroser G (Biosepra, Cedex-Saint-Christophe, France), and glucose $(4.5 \mathrm{mg} / \mathrm{ml})$ [1,17-19]. For induction of myogenic differentiation, the cells were plated and cultured for $24 \mathrm{~h}$ in pmGM, and then the medium was changed to pmDM consisting of the chemically defined medium TIS [20,21] supplemented with $2 \%$ FBS. A Ric10-derived clone constitutively expressing GFP-GPI, GGS25 [10], was cultured under the same conditions as Ric10. The Src kinase inhibitor SU6656 and Rac1 inhibitor NSC23766 (Sigma, St. Louis, MO) were dissolved in dimethylsulfoxide and diluted with culture medium immediately before use.

\section{Transfection}

Ric10 cells $\left(2 \times 10^{4}\right.$ cells in a $35-\mathrm{mm}$ dish) were transfected with $0.9 \mu \mathrm{g}$ of pcDNA-GFP-Rac1wt, GFP-Rac1DA, and GFP-Rac1DN (kindly provided by K. Kaibuchi, Nagoya University) in the presence of $4.5 \mu \mathrm{l}$ of FuGENE6 transfection reagent (Roche Diagnostic, Mannheim, Germany) as previously described [20-22].

\section{Immunofluorescence analyses}

Cells were grown on collagen-coated culture dishes, then fixed, permeabilized, and processed for immunostaining as described [1,5]. Primary antibodies included mouse monoclonal antibodies to sarcomeric myosin heavy chain (MyHC) (MF20; undiluted culture supernatant) [23], M-cadherin (1:250 dilution; BD Biosciences, San Jose, CA), p120 catenin (1:1000 dilution; BD), $\beta$-tubulin (1:100 dilution; Abcam, Cambridge, UK), flotillin (1:500 dilution; $\mathrm{BD})$, rabbit polyclonal antibodies to tyrosine phosphorylated p120 (phospho Y228) (1:500; Abcam), GFP (1:500 dilution, Medical Biological Laboratory, Nagoya, Japan). Secondary antibodies included Cy3-labeled antibodies to mouse or rabbit immunoglobulin G (1:1000 dilution; Jackson ImmunoReseach Laboratory) and Alexa Fluor 488-labeled antibodies to mouse or rabbit immunoglobulin G (1:1000 dilution; Jackson ImmunoReseach Laboratory). Cell nuclei were stained with 2,4-diamidino-2-phenylindole dihydrochloride $n$-hydrate (DAPI) $\left(0.5 \mu \mathrm{g} \mathrm{ml}{ }^{-1}\right.$, Sigma). Samples were visualized using an inverted microscope (model IX71; Olympus, Tokyo, Japan) and a CCD camera (DP70; Olympus). Images were post-processed using Adobe Photoshop (Adobe Systems, San Jose, CA).

\section{Immunoblotting and immunoprecipitation}

Sample preparation and immunoblot analyses were performed as described $[21,22,24]$. Immune complexes were detected by colorimetry with a BCIP/NBT detection kit (Sigma). Immunoprecipitation was done with a Pierce Crosslink Magnetic IP/Co-IP Kit (Thermo Fisher Scientific Inc., Rockford, IL). Threonine phosphorylation of p120 was detected by rabbit polyclonal antibodies to threonine phosphorylated p120 (phospho T310) (1:500 dilution; Abcam).

\section{Time-lapse recording}

Cells were cultured in neutral red-depleted pmDM and placed in a humid chamber (Tokai Hit, Fujinomiya, Japan) maintained at $37^{\circ} \mathrm{C}$ under $10 \% \mathrm{CO}_{2}$. Time-lapse images were taken using an inverted microscope (BZ9000; Keyence, Osaka, Japan) with a $10 \times$ or $20 \times$ Plan Apo Fluor objective lens (Nikon, Tokyo, Japan).

\section{Quantification of muscle cell hypertrophy}

The distribution of myogenic cell sizes was determined by calculating the percentage of nuclei in myogenic cells with 
different numbers of nuclei in the total number of nuclei (myoblasts plus myotubes), as described previously [5].

\section{Fractionation of detergent-resistant membranes}

Ric10 cells were cultured for $24 \mathrm{~h}$ in pmGM and then further incubated for $24 \mathrm{~h}$ in pmDM. The cells were lysed in $0.2 \mathrm{ml}$ of ice-cold lysis buffer $(0.5 \%$ Trion X-100, $50 \mathrm{mM}$ MES (pH 6.0), $50 \mathrm{mM} \mathrm{NaCl}, 5 \mathrm{mM} \mathrm{MgCl}_{2}$, and $2.5 \mathrm{mM}$ EGTA) containing protease inhibitor (Complete Protease Inhibitor Cocktail EDTA-free; Roche, Mannheim, Germany) for $30 \mathrm{~min}$ on ice. Protein concentrations in aliquots of cell lysates were determined using a BCA kit (Sigma). An aliquot of the lysate containing approximately 300-400 $\mu$ g protein was mixed with OptiPrep (Axis-Shield, London, UK) and fractionated in a $3 \mathrm{ml}$ Optiprep gradient according to the manufacturer's instructions (Caveolae/ Rafts Isolation Kit; Sigma). Ten fractions were collected from the top, and $30 \mu \mathrm{l}$ of each fraction was analyzed by immunoblotting. The PVDF membranes were scanned, and the signal intensity of each band was quantified using Image J software $(\mathrm{NIH})$. Detergent-resistant membrane (DRM) fractions consist of lipid rafts. The distribution of the protein in each fraction was determined by calculating the ratio of the signal intensity of the protein band in each fraction to the sum of the signal intensity in all fractions. In the indicated experiments, the amounts of M-cadherin and p120 in lipid rafts were quantified using Flotillin-1 as a standard. To detect the raft-specific ganglioside GM1 in DRM, $30 \mu$ of each fraction was spotted on a nitrocellulose membrane, probed with HRP-conjugated cholera toxin B subunit (CTB), then detected by colorimetry using a Fast DAB kit (Sigma). The distribution of GM1 in each fraction was determined by calculating the ratio of the signal intensity of the spot in each fraction to the sum of the signal intensity in all fractions.

\section{Results \\ M-cadherin is recruited to lipid rafts during myogenic differentiation}

Dynamic clustering and dispersion of lipid rafts at the leading edge of lamellipodia and membrane ruffles that contain presumptive fusion sites is critical for myogenic cell fusion [10]. We focused on a molecular mechanism underlying recruitment of $\mathrm{M}$-cadherin to the presumptive fusion site. GGS25 [10] is a mouse myogenic cell line that constitutively expresses GPI-anchored GFP (GFP-GPI) as a raft marker. Cells were cultured at low cell density to avoid cell-cell contact. Subcellular fractionation of GGS25 cells by density gradient ultracentrifugation showed that most M-cadherin was located at non-raft membranes in growing myogenic cells (Figure 1A). M-cadherin was recruited to lipid rafts under the terminal muscle differentiation-inducing condition, whereas the concentrations of raft marker molecules, flotillin and ganglioside

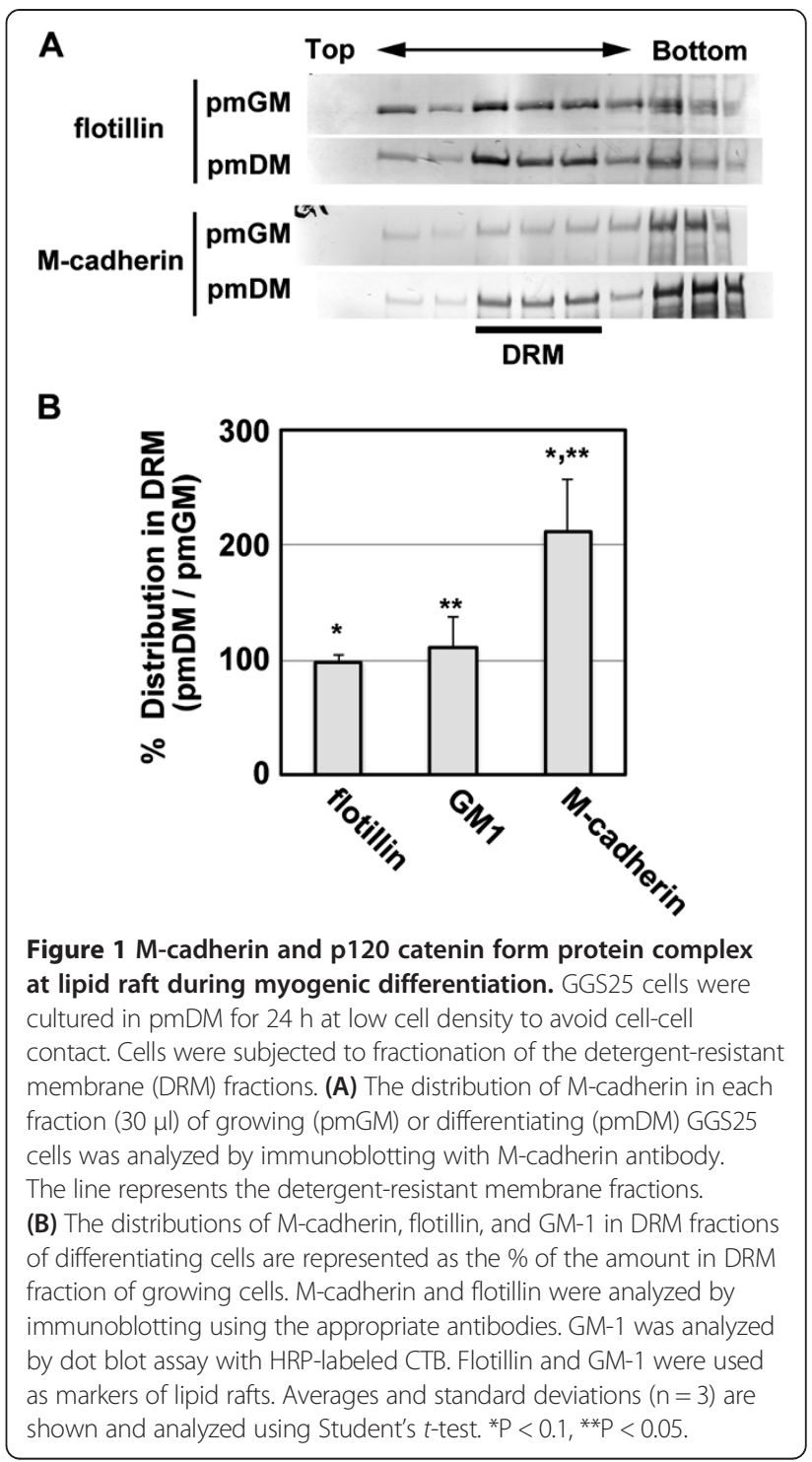

GM1, remained constant during myogenesis (Figure 1A, B). The results were consistent with the previous observation showing that $\mathrm{M}$-cadherin is accumulated at lipid rafts in cell contact-free regions of plasma membrane [10].

\section{Active Rac1 enhances myogenic cell fusion}

The small G protein Rac1 is known to play a pivotal role in lamellipodium formation in various cell types. Rac1 physically interacts with M-cadherin [25] and is activated by $\mathrm{p} 120[26,27]$ and M-cadherin [25]. Therefore, we determined whether Rac1 is involved in the recruitment of M-cadherin to lipid rafts during myogenic cell fusion.

Expression plasmids encoding GFP-fused wild (WT), constitutively active (CA), or dominant negative (DN) Rac1 were transfected into Ric10 cells under the differentiationinducing condition to avoid the inhibitory effects of Rac1 
on the initiation of myogenesis [28,29]. Therefore, MyHC was expressed in $97.0 \%, 94.0 \%$, or $96.3 \%$ of Ric10 cells transfected with GFP-Rac1WT, CA, or DN expression plasmids, respectively (Figure 2A). Fusion indexes were $89.0 \%$, 91.1\%, or $73.6 \%$ in Ric10 cells expressing Rac1WT, CA, or DN, respectively. In addition, Rac1CA-expressing Ric10 cells gave rise to large multinucleated cells called myosheets [5] containing more than 31 nuclei (Figure 2 middle panels in A and B). It suggests that Rac1CA promotes cell fusion between myotubes or myoblasts and myotubes as well as myoblasts. In contrast, Ric10 cells expressing Rac1DN formed small myotubes exclusively (Figure 2 right-hand panels in $\mathrm{A}$ and $\mathrm{B}$ ). The results indicated that Rac1 promotes myogenic cell fusion depending on its activity.

Rac1 activity is required for microraft organization at presumptive fusion sites

GFP-Rac1DN prevented lamellipodium formation and often induced a severely abnormal morphology in Ric10 cells

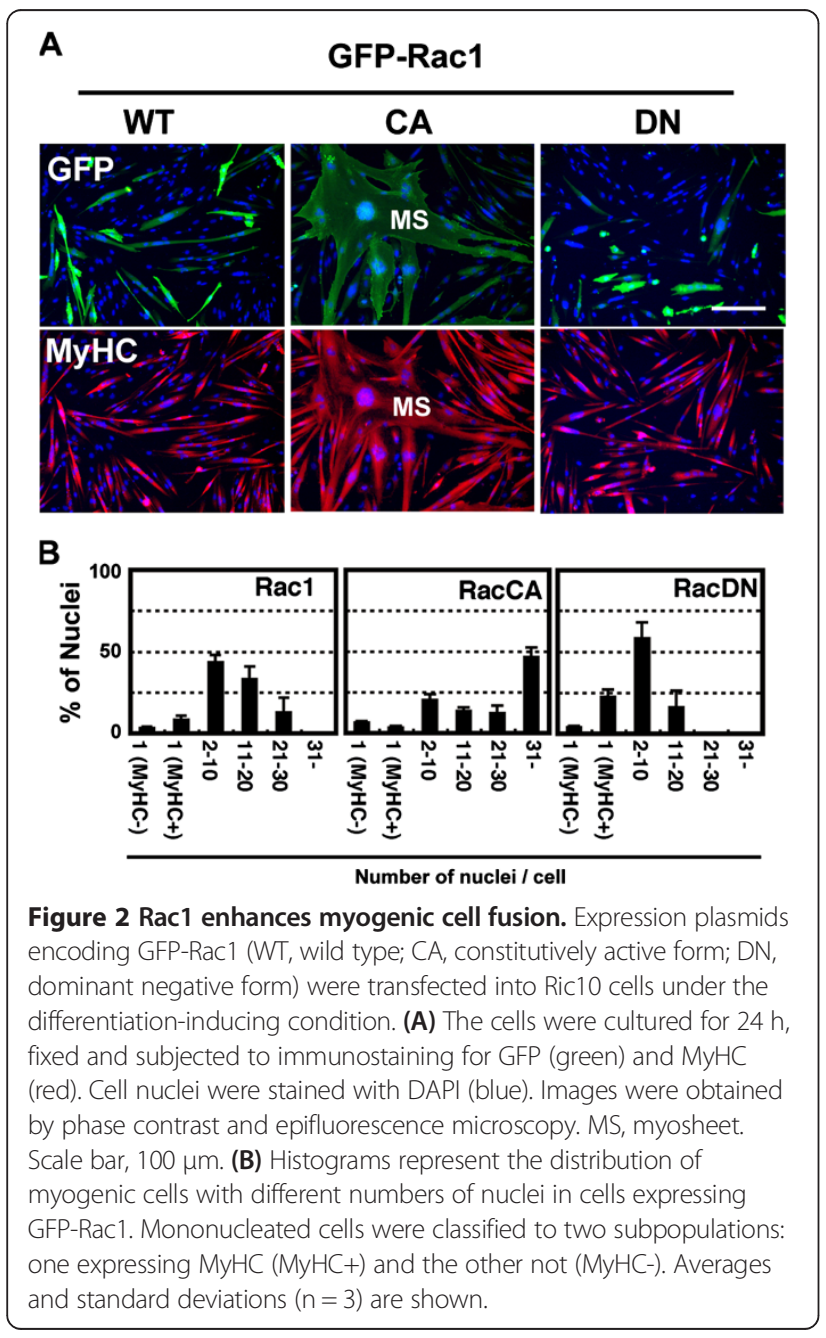

(data not shown), implying that it produced unphysiologically severe damage to the cells, perhaps due to the disordered arrangement of cytoskeletons. Therefore, we used the Rac1 inhibitor NSC23766 to inhibit Rac1 activity to more moderate levels. NSC23766 did not inhibit the expression of $\mathrm{MyHC}$ at $100 \mu \mathrm{M}$ (Figure 3A), although it attenuated myogenesis of Ric10 at $200 \mu \mathrm{M}$ (data not shown). However, $100 \mu \mathrm{M}$ NSC23766 inhibited myogenic cell fusion without compromising the expression of $\mathrm{MyHC}$ (Figure 3A), as previously described in $\mathrm{C} 2 \mathrm{C} 12$ cells [25].

To determine the role of Rac1 in microraft organization, GGS25 cells expressing GFP-GPI were treated with NSC23766. Lipid rafts were visualized in living cells by GFP-GPI under an epifluorescence microscope. Microrafts were rarely organized under the growing condition (Figure $3 \mathrm{~B}$ and Additional file 1), but organization of microrafts was markedly promoted at membrane ruffles under the differentiation-inducing condition (Figure 3C and Additional file 2). Intensive GFP fluorescence at membrane ruffles of differentiating GGS25 cells showed dense clusters produced by a large number of nanometer-sized lipid rafts. In contrast, microraft organization was severely suppressed in differentiating GGS25 cells treated with NSC23766 (Figure 3D and Additional file 3).

To quantify the inhibitory effects of NSC23766 on microraft organization, a microraft visualized by GFP-GPI was observed by time-lapse recording for $1 \mathrm{~h}$ from the indicated time in Figure 3E. The number of cells that organized microrafts at membrane ruffles markedly increased under the differentiation-inducing condition (Figure $3 \mathrm{C}$ and $\mathrm{E}$ ). The frequency of microraft organization at lamellipodia of an individual cell also increased (Additional files 1 and 2). In contrast, NSC23766 prevented microraft organization at membrane ruffles (Figure 3D and $\mathrm{E})$. The frequency of microraft organization at lamellipodia severely declined in NSC23766-treated cells compared to that of untreated cells (Additional file 3). These results showed that Rac1 plays a pivotal role in microraft formation at the presumptive fusion site and suggest that organization of microraft is essential for myogenic cell fusion.

Rac1 activity is dispensable for recruitment of M-cadherin to lipid rafts and interaction with p120 catenin

In the next series of experiments, we determined whether a Rac1-dependent organization of microrafts is required for differentiation-induced recruitment of $\mathrm{M}$-cadherin to lipid rafts. The plasma membrane was fractionated by density gradient ultracentrifugation. NSC23766 did not affect the amounts of $\mathrm{M}$-cadherin and raft markers flotillin and GM1 in the raft fraction (Figure 4A). Furthermore, similar concentrations of p120 were found in immunoprecipitates from both control and NSC23766-treated Ric10 

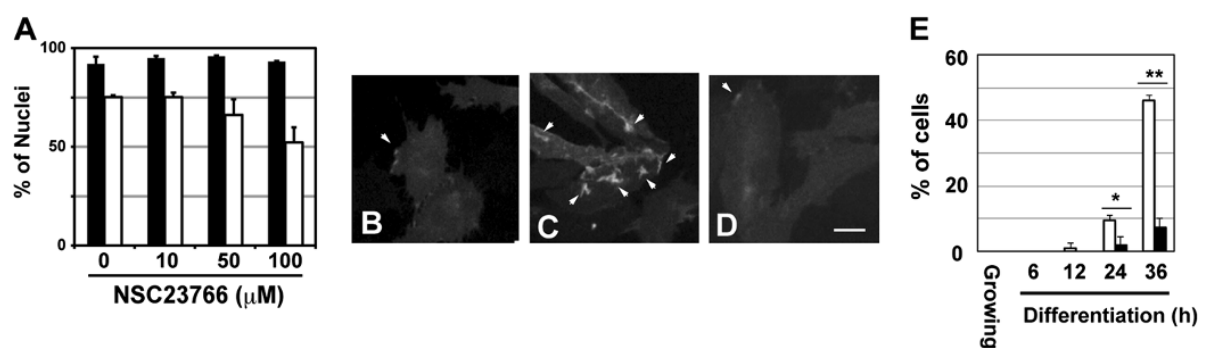

Figure 3 Rac1 inhibitor suppresses microraft organization during myogenic differentiation. (A) GGS25 cells were cultured for $24 \mathrm{~h}$ and then further cultured in pmDM supplemented with 0.1\% DMSO or NSC23766 (10-100 $\mu \mathrm{M})$ for up to $36 \mathrm{~h}$. Differentiated cells were detected by immunostaining with anti-MyHC antibody (filled columns). Fusion indexes were calculated as percentages of nucleus numbers in multinucleated cells (open columns). Averages and standard deviations $(n=3)$ are shown. (B-E) GGS25 cells were cultured in pmGM (B), pmDM with 0.1\% DMSO (C and E), or pmDM with NSC23766 (100 $\mu \mathrm{M})$ for up to $37 \mathrm{~h}$ (D and E). GFP-GPI was sequentially observed at 1 min intervals under epifluorescence microscopy by time-lapse recording. Pictures were taken after $24 \mathrm{~h}$ of differentiation cultures (C and D) or growing culture (B). Scale bar, $25 \mu \mathrm{m}$. (E) Percentage of cells that showed membrane ruffles during $1 \mathrm{~h}$ following the indicated time in pmDM supplemented with DMSO (open columns) or NSC23766 (filled columns). Averages and standard deviations $(n=3)$ are shown and analyzed using Student's t-test. ${ }^{* * *} P<0.05$.

cells with anti-M-cadherin antibody (Figure 4B). Thus, microraft organization might be regulated independently of the recruitment of M-cadherin and p120 to nanometersized lipid rafts and their physical interaction.

\section{M-cadherin interacts with $\mathrm{p} 120$ catenin predominantly at lipid rafts during myogenic differentiation}

Cell contact-independent recruitment of adhesion molecules to lipid rafts is likely to be a critical step of myogenic cell fusion [10]. P120 catenin is a probable candidate for a key molecule in recruitment of $\mathrm{M}$-cadherin because it regulates the locations of $\mathrm{N}$-cadherin in lipid rafts [30]. The amounts of M-cadherin increased prior to the

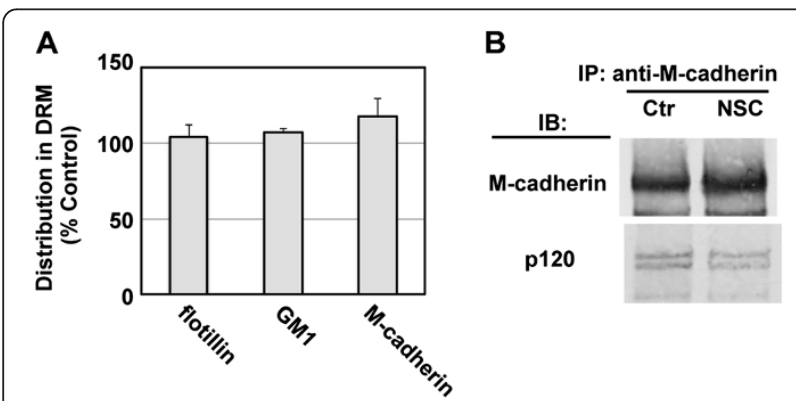

Figure 4 Interaction between $\mathrm{p} 120$ and M-cadherin is independent of Rac1 activity. Ric10 cells were cultured in pmGM for $24 \mathrm{~h}$ and then further cultured in pmDM for up to $24 \mathrm{~h}$. (A) Plasma membrane was fractionated by density-gradient ultracentrifugation. DRM fractions were pooled and subjected to immunoblot analysis for flotillin and M-cadherin and dot blot analysis for GM-1. The distribution of molecules in DRM fractions of NSC23766-treated cells is represented as the \% of the amount in DRM fraction of untreated control cells. Averages and standard deviations ( $n=3)$ are shown. (B) Ric10 cells were cultured for $24 \mathrm{~h}$ in pmDM supplemented with $0.1 \%$ DMSO (Ctrl) or 100 MM NSC23766 (NSC). Then, immunoprecipitated materials from total cell lysates were subjected to immunoblot analysis. Similar results were obtained by two independent experiments. Representative results were shown. expression of the muscle-differentiation marker myosin heavy chain (MyHC) (Figure 5A). The amounts of p120 catenin increased slightly. In addition, a specific antibody against the tyrosine phosphorylated form of p120 revealed that its phosphorylation was robustly enhanced during myogenic differentiation (Figure 5A). Phosphorylation of threonine residues of p120 was also enhanced. Co-immunoprecipitation assays indicated that physical interaction between $\mathrm{M}$-cadherin and p120 was promoted during myogenic differentiation (Figure 5B). In addition, the complex including M-cadherin and p120 catenin was found predominantly in lipid rafts (Figure 5C). The results suggest that $\mathrm{M}$-cadherin is associated with p120 in a lipid raft-dependent fashion during myogenic differentiation.

To determine the role of Src family kinases in interaction of p120 with M-cadherin, Ric10 cells were treated with the Src kinase inhibitor SU6656 because Src family kinases are major protein kinases that phosphorylate tyrosine residues of p120 [31]. SU6656 markedly attenuated the interaction between p120 and M-cadherin (Figure 5D). Furthermore, the amounts of M-cadherin and p120 at lipid rafts declined in SU6656-treated cells (Figure 5E). Recruitment of Ncadherin to lipid rafts was also attenuated by SU6656 in a manner similar to M-cadherin (data not shown). SU6656 attenuated physical interaction between $\mathrm{M}$-cadherin and p120 catenin, and their recruitment to lipid rafts, implicating the role of Src family kinases in the regulation of recruitment of $\mathrm{M}$-cadherin to lipid rafts.

\section{Src kinase inhibitor suppresses myogenic cell fusion without compromising myogenic differentiation}

Recruitment of M-cadherin to lipid rafts is supposed to be indispensable for its accumulation at the presumptive fusion site [10]. Actually, SU6656 suppressed myogenic cell fusion in a dose-dependent manner (Figure 6A-D, F and $G$ ), although it did not inhibit expression of the 

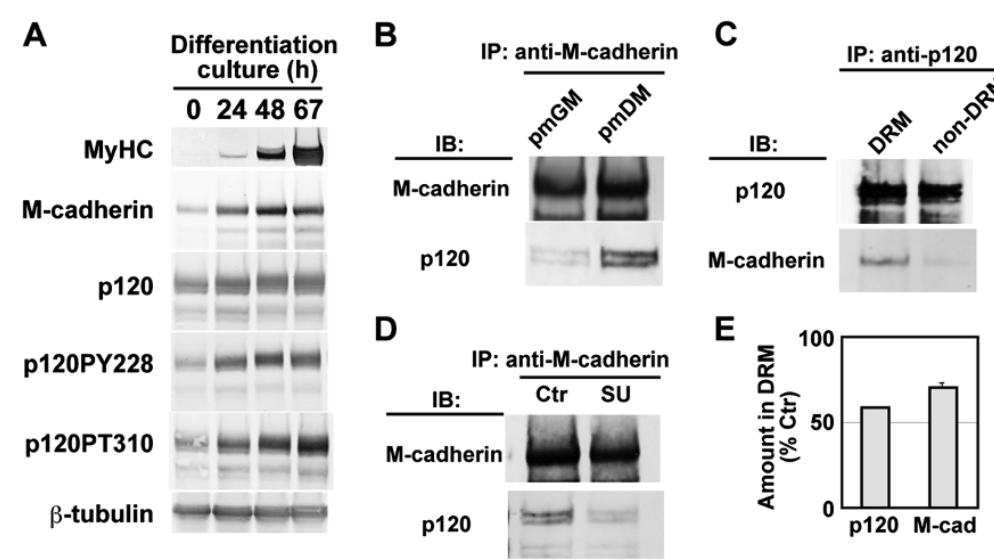

Figure 5 Src kinase inhibitor impairs physical interactions between M-cadherin and p120 catenin and their recruitment to lipid rafts. (A) Total lysates (20 $\mathrm{\mu g}$ of proteins) were prepared from Ric10 cells cultured in pmDM for $0 \mathrm{~h}$ (lane 1), $24 \mathrm{~h}$ (lane 2), $48 \mathrm{~h}$ (lane 3), or $67 \mathrm{~h}$ (lane 4), and then subjected to immunoblot analysis for the represented proteins. MyHC, myosin heavy chain; P120PY228, tyrosine phosphorylated p120; p120PT310, threonine phosphorylated p120. $\beta$-tubulin was used as a loading control. (B) M-cadherin of growing or differentiating Ric10 cells was immunoprecipitated and analyzed by immunoblotting with the appropriate antibodies. (C) p120 catenin was immnoprecipitated from the DRM or non-DRM fractions of differentiating Ric10 cells, and then analyzed by immunoblotting with the appropriate antibodies. (D) M-cadherin was immunoprecipitated from total cell lysates of untreated (Ctrl) or SU6656-treated Ric10 cells (SU), and then analyzed by immunoblotting with the appropriate antibodies. (E) The distribution of M-cadherin and p120 catenin in DRM fractions (30 $\mu$ l) of untreated or SU6656-treated Ric10 cells was analyzed by immunoblotting with the appropriate antibodies. The distribution of M-cadherin and p120 catenin in DRM was estimated as the relative amount normalized by the amount of flotillin in DRM fractions. Then the distribution of M-cadherin and p120 in DRM fractions of SU6656-treated cells was represented as the \% of the amount in DRM fraction of untreated control cells. Averages and standard deviations $(n=3)$ are shown.

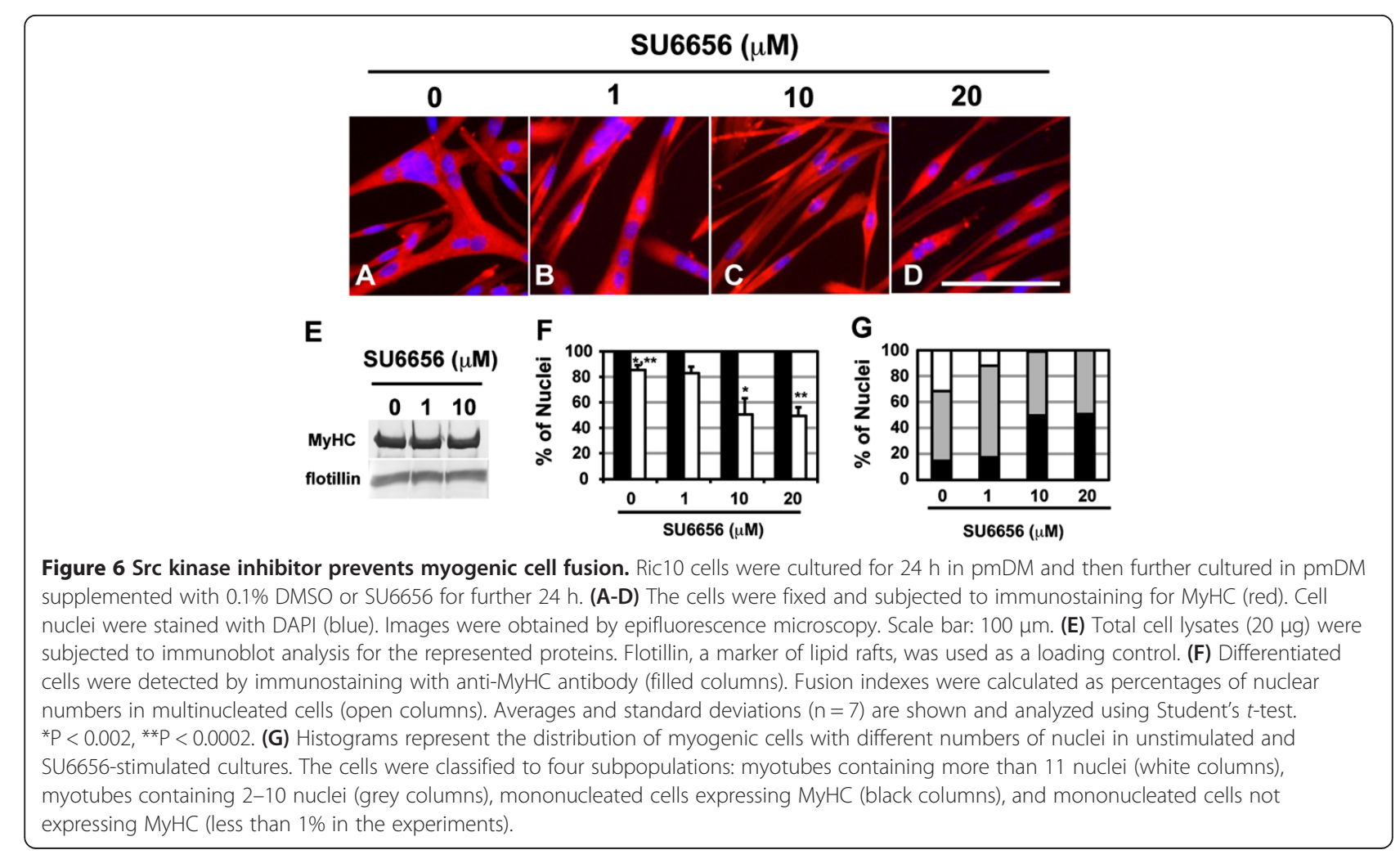


myogenic differentiation marker MyHC (Figure 6A-F), M-cadherin, or p120 (Additional file 4A). Inhibition of cell fusion by SU6656 resulted in a severe reduction in the number of larger myofibers containing more than 11 nuclei (Figure 6G).

\section{Src kinase inhibitor suppresses accumulation of tyrosine- phosphorylated p120 and organization of microrafts}

Despite the robust inhibition by SU6656 of recruitment of M-cadherin to lipid rafts and myogenic cell fusion, antibodies recognizing phosphorylated tyrosine residue 228 of p120 did not show a decline in the amounts of tyrosine phosphorylated p120 in the total cell lysate (Additional file 4A). In addition, the amounts of tyrosine phosphorylated p120 increased when treated with the tyrosine phosphatase inhibitor vanadate (Additional file 4B). Thus, turnover of phosphorylated tyrosine residues of p120 might depend predominantly on tyrosine phosphatase. The results suggest that other Src kinase substrates are involved in physical interaction between M-cadherin and p120 catenin, and their recruitment to lipid rafts.

It is likely that subcellular localization of p120 is critical to myogenic cell fusion. Therefore, the effects of SU6656 on the subcellular distribution of tyrosine-phosphorylated p120 in differentiating myogenic cells were determined. Immunostaining analyses showed that tyrosine phosphorylated p120 accumulated at membrane ruffles (Figure 7Aa-h). SU6656 inhibited the distribution of p120 at membrane ruffles (Figure $7 \mathrm{Ai}-\mathrm{l})$. Thus, Src family kinases might induce the redistribution of p120 at membrane ruffles through phosphorylation of other substrates. In contrast, p120 accumulated at cell contacts even in SU6656-treated cells (Figure 7Am-p) as well as in control cultures (Additional file 5).

To determine whether SU6656 inhibits membrane ruffling in differentiating myogenic cells, Ric10 cells were sequentially observed by time-lapse recording. The plasma membrane of myogenic cells frequently ruffled during myogenic differentiation (Figure 7B top row and Additional file 6). SU6656 prevented membrane ruffling within 10 minutes after administration (Figure 7B second row and Additional file 7). The tyrosine phosphatase inhibitor vanadate did not affect membrane ruffling of Ric10 cells (Figure 7B third row and Additional file 8). However, a 20min pretreatment with a high concentration of vanadate $(100 \mu \mathrm{M})$ partially but clearly antagonized the inhibitory effect of SU6656 on membrane ruffling of Ric10 cells (Figure 7B bottom row and Additional file 9). The results suggest that tyrosine phosphorylation under the control of Src kinase is involved in the regulation of membrane ruffling.

To obtain direct evidence that SU6656 affects the organization of microrafts, GFP-GPI in GGS25 cells was sequentially observed by time-lapse recording. Rapid cycles of clustering and dispersion of microrafts were visualized by GFP-GPI under epifluorescence microscopy (Figure 7C upper panels and Additional file 10). In contrast, SU6656 perfectly suppressed the organization of microrafts (Figure 7C lower panels and Additional file 11).

Taken together with the results above, it is suggested that Src kinase plays a critical role in recruitment of M-cadherin/p120 to lipid rafts. In addition, the results imply that the recruitment of adhesion proteins to lipid rafts might be relevant to microraft organization at the presumptive fusion site.

\section{Discussion}

Dynamic clustering and dispersion of lipid rafts is required to establish fusion-competent sites on the myogenic cell membrane [10]. Although cell adhesion and membrane fusion are sequential steps in myogenic cell fusion, both steps require distinct microcircumstances in the plasma membrane. To establish cell adhesion, the plasma membrane at the presumptive fusion site must contain enough cholesterol to maintain the rigid lipid bilayers that hold adhesion complexes. However, membrane fusion takes place at cholesterol-free spots of the plasma membrane $[11,32,33]$, and adhesion complexes are removed from the fusion site prior to membrane breakdown/union [10]. The dynamic clustering and dispersion of lipid rafts enables robust and rapid changes in plasma membrane components at the presumptive fusion site. However, the pre-fusion events required for specialization of fusion-competent sites have been unknown. The present study showed that both the recruitment of adhesion complexes to lipid rafts and the organization of microrafts are required for establishing fusion-competent sites.

One possible mechanism of M-cadherin recruitment to lipid rafts could be based on control of its binding with p120 catenin. P120 is essential for stability of cadherins on the plasma membrane in a direct interaction-dependent manner [34,35]. Tyrosine-phosphorylated p120 shows increased affinity to cadherins [36-39]. Tyrosine phosphorylation of p120 also requires recruitment of p120 to plasma membranes [31,40,41]. In addition, only membraneassociated Src can phosphorylate p120 [42]. However, we were unable to detect a significant difference in the total amounts of tyrosine-phosphorylated p120 between control and Src kinase inhibitor SU6656-treated differentiating myogenic cells. A major fraction of tyrosine-phosphorylated p120 retained the phosphorylated state even when Src family kinases were inhibited. Despite that, SU6656 inhibited differentiation-induced recruitment of M-cadherin/p120 to lipid rafts and their physical interaction. Thus, in the physiological cellular context, another Src kinase substrate might play a role in the recruitment of M-cadherin/p120 to lipid rafts. Actually, Src kinase stimulates the E-cadherin regulator protein to regulate cell-cell adhesion [43]. 


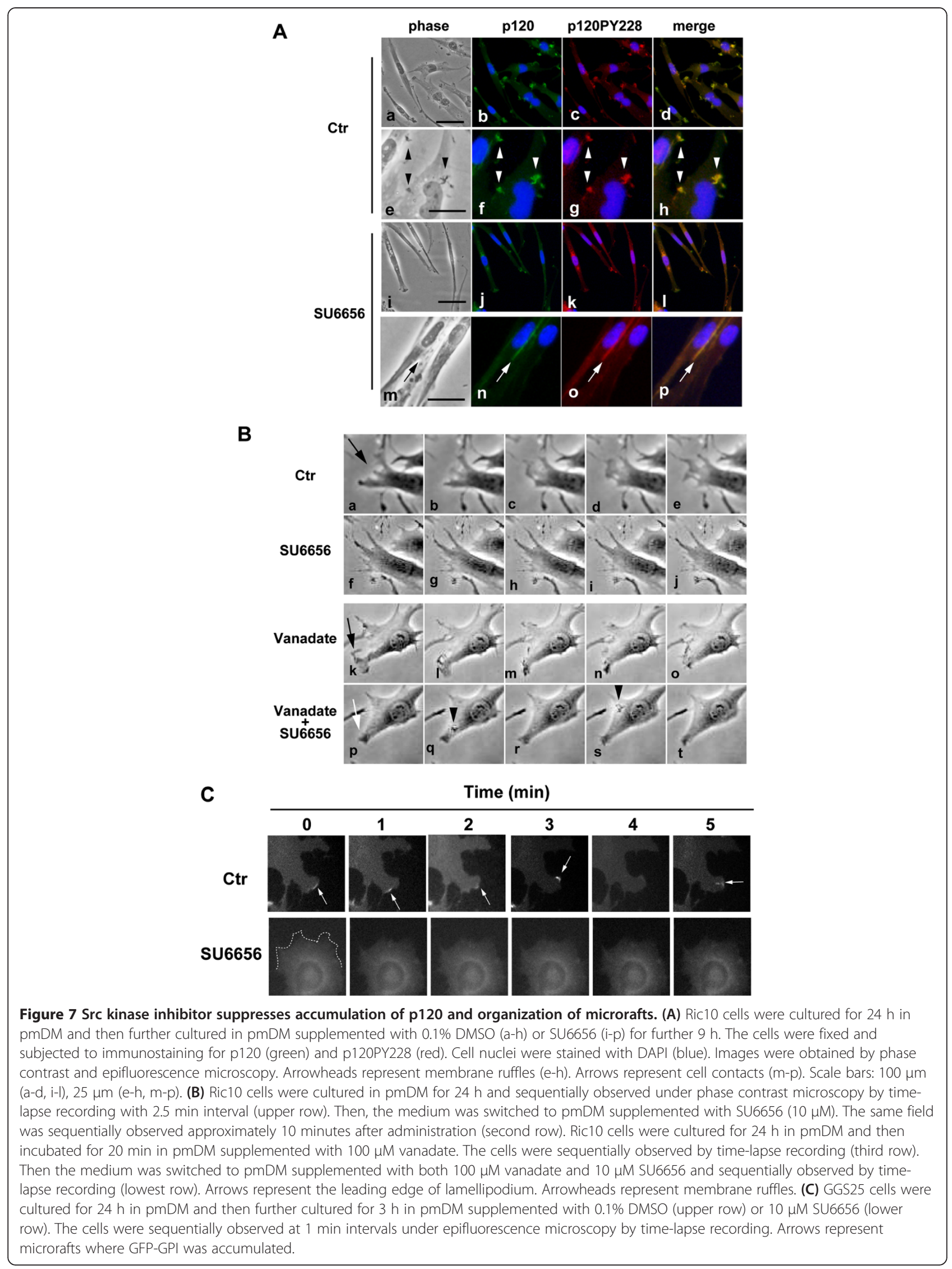


Crosstalk between cAMP-dependent protein kinase (PKA) and Src pathways $[44,45]$ is another possible mode of Src kinase action for M-cadherin recruitment because the localized PKA pathway is involved in the specialization of the fusion-competent areas of the plasma membrane in myogenic cells [5].

SU6656 prevented both microraft organization and membrane ruffling. Src family kinases bind to the autophosphorylated focal adhesion kinase (FAK) that is activated by integrin-mediated adhesion [46]. The active FAK-Src complex stimulates Rac1 activity through phosphorylation of a number of mediators including the scaffolding protein p130Cas, paxillin, paxillin kinase linker, Pak-interacting exchange factor-beta, and spleen tyrosine kinase. Thus, Src is likely to play a role in microraft organization through stimulation of Rac1 activity.

P120 catenin also modulates the activity and spatial distribution of Rac1 [25,47-49]. Either SU6656 or the Rac1 inhibitor NSC23766 inhibited the organization of microrafts, the generation of membrane ruffles/lamellipodia, and cell fusion of myogenic cells under the differentiation-inducing condition. We were unable to detect a significant difference between control and SU6656treated cells in the total Rac1 activity by a pull-down assay. However, we did see effects of SU6656 on differentiating myogenic cells similar to those of NSC27366. The p120knockdown inhibits the lamellipodia dynamics and localization of Rac1 but doesn't decrease the total Rac1 activity [49]. SU6656 might modulate the spatial distribution of Rac1, as shown in p120 knockdown cells.

Subcellular distribution of cadherins is determined by at least two distinct dynamic cycles: the trans-directional membrane-cytoplasmic transport/endocytosis cycle [50] and the cis-directional lateral clustering/dispersion cycle within the plasma membrane $[10,51,52]$. The underlying mechanism controlling the recruitment of cadherin to lipid rafts remained to be discerned, while relevant factors, including clathrin and p120 catenin, have been proposed for regulation of the membrane transport/endocytosis cycle $[53,54]$. In the cis-cycle, the distribution of M-cadherin in lipid rafts or non-raft regions of the plasma membrane corresponds to a dynamic equilibrium of association and dissociation with $\mathrm{p} 120$. The accumulation of M-cadherin at microrafts is found at both cell contact and presumptive fusion sites of myogenic cells [10]. The present study shows that M-cadherin is recruited to lipid rafts independently of the microraft organization. The previous and present studies imply that the functional M-cadherin/p120 catenin complex is first recruited to nanometer-sized lipid rafts (nanorafts). Then, either cell-cell contact or myogenic differentiation induces nanorafts to give rise to a microraft, resulting in the robust accumulation of cadherin/p120 complexes at the cell contact and presumptive fusion site (Figure 8).

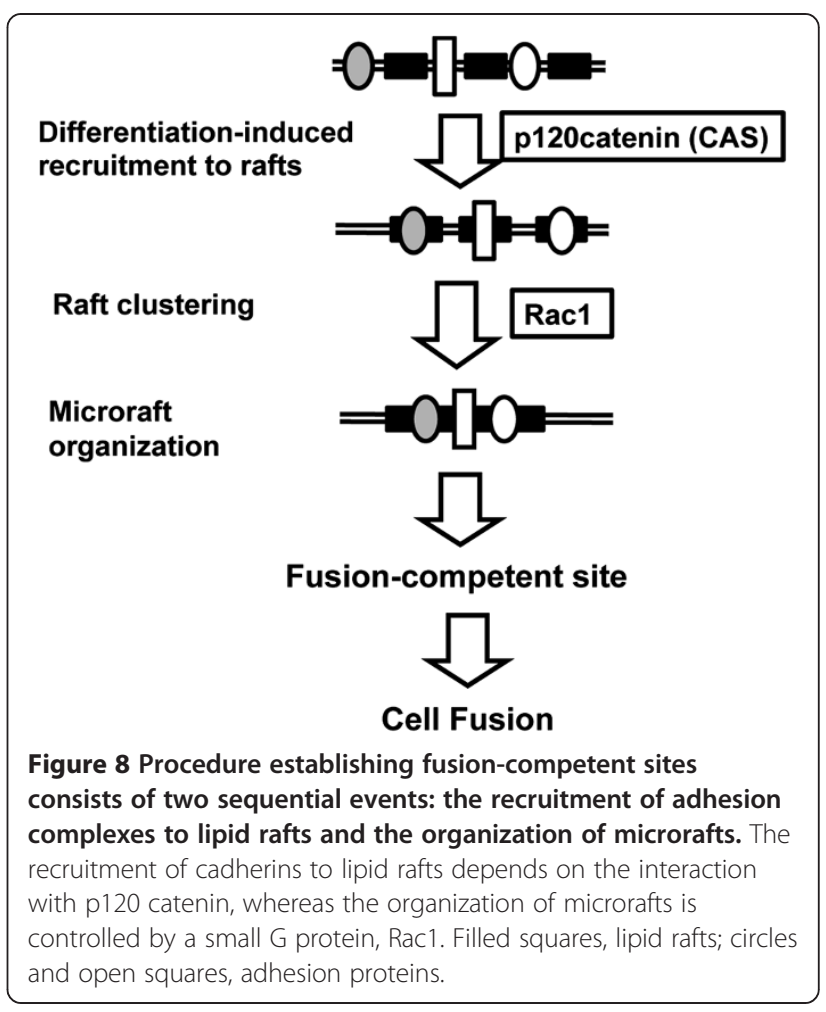

$\mathrm{N}$-cadherin as well as $\mathrm{M}$-cadherin plays a role in myogenic cell fusion [55]. N-cadherin also accumulates at microrafts at ruffling membranes and the leading edge of lamellipodia of differentiating myogenic cells [10]. P120 is involved in the regulation of N-cadherin location in lipid rafts [30]. In addition, the present study shows that SU6656 also suppressed the recruitment of $\mathrm{N}$-cadherin to lipid rafts. Taken together, the recruitment of $\mathrm{N}$-cadherin and $\mathrm{M}$-cadherin to lipid rafts might have common mechanisms in differentiating myogenic cells.

Cadherins are involved in cell recognition and adhesion by homophilic interactions conferred by their extracellular regions. Their intracellular regions link them with cytoplasmic partner proteins and consequently the actin filament network. Thus, our findings on the accumulation of cadherins at the presumptive fusion sites might be related to remodeling of the actin cytoskeleton. We previously analyzed the arrangement of F-actin during myogenic cell fusion $[5,10]$. Briefly, cadherin complex is co-aligned with F-actin at ruffling membranes and the leading edge of lamellipodia. The cortical actin cytoskeleton at presumptive fusion sites might play a critical role in lateral dispersion of lipid rafts. Interestingly, after myogenic cell fusion, both the ruffling membrane and lamellipodium disappear except at the polar ends of myotubes [5]. The localized accumulation of cadherins at the polar ends of myotubes might create anchoring points of actin filaments and contribute to remodeling actin cytoskeleton during myogenic differentiation. 
The present results suggest that the recruitment of M-cadherin/p120 complex to lipid rafts of cell contact - free surfaces is essential for the specialization of fusion-competent areas of the plasma membrane. Src kinase activity is likely to be critical to the recruitment of M-cadherin to lipid rafts. Rac1 induces the dynamic organization of microrafts at the presumptive fusion site. However, the mechanism connecting these two sequential events has not been discerned. It is likely that Rac1 is activated downstream of Src kinase and M-cadherin/p120 during myogenic differentiation [25,46-48]. The present study provides a possible molecular mechanism underlying the specialization of presumptive fusion sites of myogenic cells.

\section{Conclusions}

Myoblast fusion consists of a series of steps including plasma membrane breakdown/union that is initially induced in a discrete area of the plasma membrane.

However, the pre-fusion events that are relevant to specialization of fusion-competent sites of the plasma membrane remained to be discerned. Here we showed that the procedure establishing fusion-competent sites consists of two sequential events: the recruitment of adhesion complexes to lipid rafts and the organization of microrafts (Figure 8). The recruitment of M-cadherin to lipid rafts depended on interaction with p120 catenin, whereas the organization of microrafts was controlled by a small G protein, Rac1.

\section{Availability of supporting data}

The data sets supporting the results of this article are included within the article and its additional files.

\section{Additional files}

Additional file 1: Rac1 inhibitor suppresses microraft organization during myogenic differentiation. GGS25 cells were cultured in pmGM. GFP-GPI was sequentially observed at 1 min intervals under epifluorescence microscopy by time-lapse recording. Many microrafts were organized in pmDM, whereas the frequency of microraft organization and the signal intensity of GFP-GPI declined in pmGM and pmDM with NSC23766.

Additional file 2: GGS25 cells were cultured in pmDM for up to $37 \mathbf{h}$. GFP-GPI was sequentially observed at $1 \mathrm{~min}$ intervals under epifluorescence microscopy by time-lapse recording. Many microrafts were organized in pmDM.

Additional file 3: GGS25 cells were cultured in pmDM with NSC23766 $(100 \mu \mathrm{M})$ for up to $37 \mathrm{~h}$. GFP-GPI was sequentially observed at $1 \mathrm{~min}$ intervals under epifluorescence microscopy by time-lapse recording. The frequency of microraft organization and the signal intensity of GFP-GPI declined in pmDM with NSC23766.

Additional file 4: Effects of Src kinase inhibitor or protein tyrosine phosphatase inhibitor vanadate on fusion-related proteins of myogenic cells. Ric 10 cells were cultured for $24 \mathrm{~h}$ in pmDM and then cultured in pmDM supplemented with $0.1 \%$ DMSO (-) or SU6656 (+) for $9 \mathrm{~h}$ (A), or vanadate $(1,10,100 \mu \mathrm{M})$ for $24 \mathrm{~h}$ (B). Total lysates $(20 \mu \mathrm{g}$ of proteins) were subjected to immunoblot analyses. MyHC, myosin heavy chain; p120PY228, tyrosine phosphorylated p120; P120PT310, threonine phosphorylated p120. $\beta$-tubulin was used as a loading control.
Additional file 5: Src kinase inhibitor doesn't suppress accumulation of $\mathrm{p} 120$ at cell contacts. Ric 10 cells were cultured for $24 \mathrm{~h}$ in pmDM and then cultured in pmDM supplemented with $0.1 \%$ DMSO (Ctrl) or SU6656 (100 $\mu \mathrm{M})$ for $24 \mathrm{~h}$. Tyrosine-phosphorylated p120 accumulated at cell-cell contacts in both control cultures (Ctrl) and SU6656- (green) and p120PY228 (red)-treated cultures. Cell nuclei were stained with DAPI (blue). Images were obtained by epifluorescence microscopy.

Additional file 6: Vanadate antagonizes the inhibitory effect of Src kinase inhibitor on membrane ruffling. Ric10 cells were cultured in pmDM for $24 \mathrm{~h}$ and sequentially observed under phase contrast microscopy by time-lapse recording. Images were recorded every $2.5 \mathrm{~min}$ by phase-contrast time-lapse microscopy. Membrane ruffling in pmDM (Additional file 6) was suppressed in pmDM supplemented with SU6656 (Additional file 7). Membrane ruffling in pmDM supplemented with vanadate (Additional file 8) was not suppressed in pmDM supplemented with SU6656 and vanadate (Additional file 9).

Additional file 7: Ric10 cells were cultured in pmDM for $24 \mathrm{~h}$ and sequentially observed under phase contrast microscopy by time-lapse recording (Additional file 6). Then, the medium was switched to pmDM supplemented with SU6656 $(10 \mu \mathrm{M})$. The same field was sequentially observed approximately 10 minutes after administration. Images were recorded every 2.5 min by phase-contrast time-lapse microscopy. Membrane rufflingwas suppressed in pmDM supplemented with SU6656.

Additional file 8: Ric10 cells were cultured for $24 \mathrm{~h}$ in pmDM and then incubated for $20 \mathrm{~min}$ in pmDM supplemented with $100 \mu \mathrm{M}$ vanadate. The cells were sequentially observed by time-lapse recording. Images were recorded every $2.5 \mathrm{~min}$ by phase-contrast time-lapse microscopy. Membrane ruffling in pmDM supplemented with.

Additional file 9: Ric10 cells were cultured for $24 \mathrm{~h}$ in pmDM and then incubated for $20 \mathrm{~min}$ in pmDM supplemented with $100 \mu \mathrm{M}$ vanadate. The cells were sequentially observed by time-lapse recording (Additional file 8). Then the medium was switched to pmDM

supplemented with both $100 \mu \mathrm{M}$ vanadate and $10 \mu \mathrm{M}$ SU6656 and sequentially observed by time-lapse recording. Images were recorded every $2.5 \mathrm{~min}$ by phase-contrast time-lapse microscopy. Membrane ruffling in pmDM supplemented with vanadate (Additional file 8) was not suppressed in pmDM supplemented with SU6656 and vanadate.

Additional file 10: Src kinase inhibitor suppresses organization of microrafts. GGS25 cells were cultured for $24 \mathrm{~h}$ in pmDM and then further cultured for $3 \mathrm{~h}$ in pmDM supplemented with $0.1 \% \mathrm{DMSO}$ or $10 \mu \mathrm{M}$ SU6656. Microrafts appeared as white spots and disappeared in control cultures (Additional file 10), whereas SU6656 prevented microraft organization and any plasma membrane movement (Additional file 11). Nothing moved in the latter file.

Additional file 11: GGS25 cells were cultured for $24 \mathrm{~h}$ in pmDM and then further cultured for $3 \mathrm{~h}$ in pmDM supplemented with $10 \mu \mathrm{M}$ SU6656. Images were recorded every min for 20 minutes by epifluorescence time-lapse microscopy. SU6656 prevented microraft organization and any plasma membrane movement. Nothing moved in the present movie.

Competing interests

The authors declare no competing interests.

\section{Authors' contributions}

AM and $\mathrm{NH}$ designed and performed experiments, provided critical reagents and tools, and wrote the manuscript. Both authors read and approved the final manuscript.

\section{Acknowledgements}

We thank K. Kaibuchi for providing GFP-Rac1 expression plasmids. We are also grateful to S. Oshima and T. Suzuki for their encouragement during the work. This study was supported by grants to NH from the Ministry of Health, Labor and Welfare of Japan. 
Received: 14 May 2013 Accepted: 22 August 2013

Published: 27 August 2013

\section{References}

1. Wada MR, Inagawa-Ogashiwa M, Shimizu S, Yasumoto S, Hashimoto N Generation of different fates from multipotent muscle stem cells. Development 2002, 129(12):2987-2995.

2. Wakelam MJ: The fusion of myoblasts. Biochem J 1985, 228(1):1-12

3. Knudsen KA, Smith L, McElwee S: Involvement of cell surface phosphatidylinositol-anchored glycoproteins in cell-cell adhesion of chick embryo myoblasts. J Cell Biol 1989, 109(4 Pt 1):1779-1786.

4. Chen EH, Olson EN: Towards a molecular pathway for myoblast fusion in Drosophila. Trends Cell Biol 2004, 14(8):452-460.

5. Mukai A, Hashimoto N: Localized cyclic AMP-dependent protein kinase activity is required for myogenic cell fusion. Exp Cell Res 2008, 314(2):387-397.

6. Fumagalli G, Brigonzi A, Tachikawa T, Clementi F: Rat myoblast fusion: morphological study of membrane apposition, fusion, and fission during controlled myogenesis in vitro. J Ultrastruct Res 1981, 75(1):112-125.

7. Tachibana I, Hemler ME: Role of transmembrane 4 superfamily (TM4SF) proteins CD9 and CD81 in muscle cell fusion and myotube maintenance. J Cell Biol 1999, 146(4):893-904.

8. Krauss RS, Cole F, Gaio U, Takaesu G, Zhang W, Kang JS: Close encounters: regulation of vertebrate skeletal myogenesis by cell-cell contact. J Cell Sci 2005, 118(Pt 11):2355-2362.

9. Horsley V, Pavlath GK: Forming a multinucleated cell: molecules that regulate myoblast fusion. Cells Tissues Organs 2004, 176(1-3):67-78.

10. Mukai A, Kurisaki T, Sato SB, Kobayashi T, Kondoh G, Hashimoto N: Dynamic clustering and dispersion of lipid rafts contribute to fusion competence of myogenic cells. Exp Cell Res 2009, 315(17):3052-3063.

11. Sekiya T, Takenawa T, Nozawa Y: Reorganization of membrane cholesterol during membrane fusion in myogenesis in vitro: a study using the filipin-cholesterol complex. Cell Struct Funct 1984, 9(2):143-155.

12. Charrasse S, Comunale F, Grumbach Y, Poulat F, Blangy A, Gauthier-Rouviere C: RhoA GTPase regulates M-cadherin activity and myoblast fusion. Mol Biol Cell 2006, 17(2):749-759.

13. Donalies M, Cramer M, Ringwald M, Starzinski-Powitz A: Expression of M-cadherin, a member of the cadherin multigene family, correlates with differentiation of skeletal muscle cells. Proc Natl Acad Sci U S A 1991, 88(18):8024-8028.

14. Kuch C, Winnekendonk D, Butz S, Unvericht U, Kemler R, Starzinski-Powitz A: M-cadherin-mediated cell adhesion and complex formation with the catenins in myogenic mouse cells. Exp Cell Res 1997, 232(2):331-338.

15. Zeschnigk M, Kozian D, Kuch C, Schmoll M, Starzinski-Powitz A: Involvement of M-cadherin in terminal differentiation of skeletal muscle cells. J Cell Sci 1995, 108(Pt 9):2973-2981.

16. Krauss RS: Regulation of promyogenic signal transduction by cell-cell contact and adhesion. Exp Cell Res 2010, 316(18):3042-3049.

17. Hashimoto N, Murase T, Kondo S, Okuda A, Inagawa-Ogashiwa M: Muscle reconstitution by muscle satellite cell descendants with stem cell-like properties. Development 2004, 131(21):5481-5490.

18. Hashimoto N, Kiyono T, Wada MR, Umeda R, Goto Y, Nonaka I, Shimizu S, Yasumoto S, Inagawa-Ogashiwa M: Osteogenic properties of human myogenic progenitor cells. Mech Dev 2008, 125(3-4):257-269.

19. Hashimoto N, Kiyono T, Wada MR, Shimizu S, Yasumoto S, Inagawa M: Immortalization of human myogenic progenitor cell clone retaining multipotentiality. Biochem Biophys Res Commun 2006, 348(4):1383-1388.

20. Hashimoto N, Ogashiwa M: Isolation of a differentiation-defective myoblastic cell line, INC-2, expressing muscle LIM protein under differentiation-inducing conditions. Dev Growth Differ 1997, 39(3):363-372.

21. Hashimoto N, Ogashiwa M, Iwashita S: Role of tyrosine kinase in the regulation of myogenin expression. Eur J Biochem 1995, 227(1-2):379-387.

22. Hashimoto N, Ogashiwa M, Okumura E, Endo T, Iwashita S, Kishimoto T: Phosphorylation of a proline-directed kinase motif is responsible for structural changes in myogenin. FEBS Lett 1994, 352(2):236-242.

23. Bader D, Masaki T, Fischman DA: Immunochemical analysis of myosin heavy chain during avian myogenesis in vivo and in vitro. J Cell Biol 1982, 95(3):763-770.

24. Hirano $\mathrm{H}$, Watanabe T: Microsequencing of proteins electrotransferred onto immobilizing matrices from polyacrylamide gel electrophoresis: application to an insoluble protein. Electrophoresis 1990, 11(7):573-580.
25. Charrasse S, Comunale F, Fortier M, Portales-Casamar E, Debant A, GauthierRouviere C: M-cadherin activates Rac1 GTPase through the Rho-GEF trio during myoblast fusion. Mol Biol Cell 2007, 18(5):1734-1743.

26. McCrea PD, Gu D: The catenin family at a glance. J Cell Sci 2010, 123(Pt 5):637-642.

27. Anastasiadis PZ: p120-ctn: A nexus for contextual signaling via Rho GTPases. Biochim Biophys Acta 2007, 1773(1):34-46.

28. Gallo R, Serafini M, Castellani L, Falcone G, Alema S: Distinct effects of Rac1 on differentiation of primary avian myoblasts. Mol Biol Cell 1999, 10(10):3137-3150.

29. Takano H, Komuro I, Oka T, Shiojima I, Hiroi Y, Mizuno T, Yazaki Y: The Rho family $\mathrm{G}$ proteins play a critical role in muscle differentiation. Mol Cell Biol 1998, 18(3):1580-1589.

30. Taulet N, Comunale F, Favard C, Charrasse S, Bodin S, Gauthier-Rouviere C: $\mathrm{N}$-cadherin/p120 catenin association at cell-cell contacts occurs in cholesterol-rich membrane domains and is required for RhoA activation and myogenesis. J Biol Chem 2009, 284(34):23137-23145.

31. Reynolds AB, Daniel J, McCrea PD, Wheelock MJ, Wu J, Zhang Z: Identification of a new catenin: the tyrosine kinase substrate p120cas associates with E-cadherin complexes. Mol Cell Biol 1994, 14(12):8333-8342.

32. Kalderon N, Gilula NB: Membrane events involved in myoblast fusion. J Cell Biol 1979, 81(2):411-425.

33. Nakanishi M, Hirayama E, Kim J: Characterisation of myogenic cell membrane: II. dynamic changes in membrane lipids during the differentiation of mouse C2 myoblast cells. Cell Biol Int 2001 , 25(10):971-979.

34. Davis MA, Ireton RC, Reynolds AB: A core function for p120-catenin in cadherin turnover. J Cell Biol 2003, 163(3):525-534.

35. Ireton RC, Davis MA, van Hengel J, Mariner DJ, Barnes K, Thoreson MA, Anastasiadis PZ, Matrisian L, Bundy LM, Sealy L, et al: A novel role for p120 catenin in E-cadherin function. J Cell Biol 2002, 159(3):465-476.

36. Calautti E, Cabodi S, Stein PL, Hatzfeld M, Kedersha N, Paolo Dotto G: Tyrosine phosphorylation and src family kinases control keratinocyte cell-cell adhesion. J Cell Biol 1998, 141(6):1449-1465.

37. Kinch MS, Clark GJ, Der CJ, Burridge K: Tyrosine phosphorylation regulates the adhesions of ras-transformed breast epithelia. J Cell Biol 1995, 130(2):461-471.

38. Papkoff J: Regulation of complexed and free catenin pools by distinct mechanisms. differential effects of Wnt-1 and v-Src.J Biol Chem 1997, 272(7):4536-4543.

39. Skoudy A, Llosas MD, Garcia De Herreros A: Intestinal HT-29 cells with dysfunction of E-cadherin show increased pp60src activity and tyrosine phosphorylation of p120-catenin. Biochem J 1996, 317(Pt 1):279-284.

40. Ohkubo T, Ozawa M: p120(ctn) binds to the membrane-proximal region of the E-cadherin cytoplasmic domain and is involved in modulation of adhesion activity. J Biol Chem 1999, 274(30):21409-21415.

41. Thoreson MA, Anastasiadis PZ, Daniel JM, Ireton RC, Wheelock MJ, Johnson KR, Hummingbird DK, Reynolds AB: Selective uncoupling of p120(ctn) from E-cadherin disrupts strong adhesion. J Cell Biol 2000, 148(1):189-202.

42. Reynolds AB, Roesel DJ, Kanner SB, Parsons JT: Transformation-specific tyrosine phosphorylation of a novel cellular protein in chicken cells expressing oncogenic variants of the avian cellular src gene. Mol Cell Biol 1989, 9(2):629-638

43. Frame MC: Newest findings on the oldest oncogene; how activated src does it. J Cell Sci 2004, 117(Pt 7):989-998.

44. Cardone L, Carlucci A, Affaitati A, Livigni A, DeCristofaro T, Garbi C, Varrone S, Ullrich A, Gottesman ME, Avvedimento EV, et al: Mitochondrial AKAP121 binds and targets protein tyrosine phosphatase D1, a novel positive regulator of src signaling. Mol Cell Biol 2004, 24(11):4613-4626.

45. Obara Y, Labudda K, Dillon TJ, Stork PJS: PKA phosphorylation of Src mediates Rap1 activation in NGF and CAMP signaling in PC12 cells. J Cell Sci 2004, 117(25):6085-6094.

46. Huveneers S, Danen EH: Adhesion signaling - crosstalk between integrins. Src and Rho. J Cell Sci 2009, 122(Pt 8):1059-1069.

47. Grosheva I, Shtutman M, Elbaum M, Bershadsky AD: p120 catenin affects cell motility via modulation of activity of Rho-family GTPases: a link between cell-cell contact formation and regulation of cell locomotion. J Cell Sci 2001, 114(Pt 4):695-707.

48. Noren NK, Liu BP, Burridge K, Kreft B: p120 catenin regulates the actin cytoskeleton via Rho family GTPases. J Cell Biol 2000, 150(3):567-580, 
49. Boguslavsky S, Grosheva I, Landau E, Shtutman M, Cohen M, Arnold K, Feinstein E, Geiger B, Bershadsky A: p120 catenin regulates lamellipodial dynamics and cell adhesion in cooperation with cortactin. Proc Natl Acad Sci U S A 2007, 104(26):10882-10887.

50. Le TL, Yap AS, Stow JL: Recycling of E-cadherin: a potential mechanism for regulating cadherin dynamics. J Cell Biol 1999, 146(1):219-232.

51. Causeret M, Taulet N, Comunale F, Favard C, Gauthier-Rouviere C: $\mathrm{N}$-cadherin association with lipid rafts regulates its dynamic assembly at cell-cell junctions in C2C12 myoblasts. Mol Biol Cell 2005, 16(5):2168-2180.

52. Seveau S, Bierne H, Giroux S, Prevost MC, Cossart P: Role of lipid rafts in E-cadherin- and HGF-R/Met-mediated entry of Listeria monocytogenes into host cells. J Cell Biol 2004, 166(5):743-753.

53. Kon S, Tanabe K, Watanabe T, Sabe H, Satake M: Clathrin dependent endocytosis of E-cadherin is regulated by the Arf6GAP isoform SMAP1. Exp Cell Res 2008, 314(7):1415-1428.

54. Yanagisawa M, Anastasiadis PZ: p120 catenin is essential for mesenchymal cadherin-mediated regulation of cell motility and invasiveness. J Cell Biol 2006, 174(7):1087-1096.

55. Mege RM, Goudou D, Diaz C, Nicolet M, Garcia L, Geraud G, Rieger F: $\mathrm{N}$-cadherin and N-CAM in myoblast fusion: compared localisation and effect of blockade by peptides and antibodies. J Cell Sci 1992, 103(Pt 4):897-906.

doi:10.1186/1471-2121-14-37

Cite this article as: Mukai and Hashimoto: Regulation of pre-fusion events: recruitment of M-cadherin to microrafts organized at fusion-competent sites of myogenic cells. BMC Cell Biology 2013 14:37.

\section{Submit your next manuscript to BioMed Central and take full advantage of:}

- Convenient online submission

- Thorough peer review

- No space constraints or color figure charges

- Immediate publication on acceptance

- Inclusion in PubMed, CAS, Scopus and Google Scholar

- Research which is freely available for redistribution 